To appear in the International Journal of Control

Vol. 00, No. 00, Month 20XX, 1-18

\title{
An Assessment on the Use of Stationary Vehicles to Support Cooperative Positioning Systems
}

\author{
Rodrigo H. Ordóñez-Hurtado, Emanuele Crisostomi and Robert N. Shorten \\ (Received 00 Month 20XX; accepted 00 Month 20XX)
}

\begin{abstract}
In this paper, we evaluate the ability of stationary vehicles (e.g., parked or temporary stopped cars) as tools to enhance the capabilities of existing cooperative positioning algorithms in vehicular networks. First, some real-world facts are provided to support the feasibility of our ideas. Then, we examine the idea in greater details in terms of the technical requirements and methodological analysis, and provide a comprehensive experimental evaluation using dedicated simulations. The routing of a drone through an urban scenario is presented as a non-traditional application case, where the benefits of the proposed approach are reflected in a better utilisation of the flight time.
\end{abstract}

Keywords: Cooperative Positioning, V2V Communications, Stationary vehicles, Parked vehicles.

\section{List of Main Acronyms}

CP Cooperative Positioning

DSRC Data Short-Range Communication

EKF Extended Kalman Filter

GNSS Global Navigation Satellite System

GPS Global Positioning Systems

GCPSO Guaranteed Convergence Particle Swarm Optimiser

HDOP Horizontal Dilution-Of-Precision

ITS Intelligent Transportation System

LOS Line-Of-Sight

MAE Mean Absolute positioning Error

RSS Radio Signal Strength

RSU Road-Side Unit

SAVG Standard Average

UWB Ultra-Wide Band

V2V Vehicle-to-Vehicle

V2I Vehicle-to-Infrastructure

V2X Vehicle-to-Vehicle/Infrastructure

VANET Vehicular Ad-hoc Network

WAVG Weighted Average

Some preliminary material was presented at the 3rd International Conference on Connected Vehicles and Expo, Austria, 2014. R. Ordóñez-Hurtado is with The Hamilton Institute, Maynooth University, Maynooth, Co. Kildare, Ireland.

E. Crisostomi is with the Department of Energy, Systems, Territory and Constructions Engineering, University of Pisa, Italy.

R. Ordóñez-Hurtado and R. Shorten are with the University College Dublin, Dublin, Ireland.

R. Shorten is also with IBM Research Ireland, Dublin, Ireland. 


\section{Introduction}

Accurate road positioning in Vehicular Ad-hoc Networks (VANETs) is viewed as a key enabler for dedicated services such as road/lane pricing, lane prioritisation for special vehicles (e.g., electric vehicles) and collision avoidance systems (1), and consequently is recognised as a key technology to enable autonomous driving $(2 ; 3)$. In this context, Cooperative Positioning (CP) is a very topical problem, viewed as a means of overcoming the drawbacks of traditional Global Navigation Satellite Systems (GNSSs) for vehicular applications, including high dependency on atmospheric/environment conditions and multipath propagation, by taking advantage of Intelligent Transportation Systems (ITSs) to collect and fuse relevant contextual data from e.g. nearby vehicles or Road-Side Units (RSUs) (4; 5).

Among the available CP techniques reported in the specialised literature, a common classification distinguishes between range-free and range-based CP systems (4). While the former realise the onroad localisation relying on support from highly dedicated Ground-Based Augmentation Systems (GBASs), such as Differential Global Positioning Systems (DGPS) and Assisted Global Positioning Systems (A-GPS) (6), the latter rely on Vehicle-to-Vehicle (V2V) and Vehicle-to-Infrastructure (V2I) ranging systems to mitigate the consequences of the low quality of GNSS signals or the nonavailability of GBASs. Whichever of the above CP approaches is chosen, its level of accuracy requires a dense deployment of dedicated infrastructure, either GBAS stations or RSUs.

The main contribution of this paper is to propose the use of stationary vehicles as an alternative to the aforementioned dedicated infrastructure, to support CP methods. In particular, we provide:

- An assessment of the feasibility of such an idea in terms of real-world supporting facts/numbers.

- A methodology to properly incorporate stationary vehicles into $\mathrm{CP}$ algorithms, based on a node-selection strategy.

- A simulated experimental study based on real-world data to evaluate the positioning accuracy of parked cars.

- A large-scale numerical evaluation and validation of the proposed strategy, in different working conditions (e.g., depending on the deployed underlying CP algorithm, ranging statistics, and positioning accuracy of parked cars).

- A non-traditional application case involving waypoint tracking for unmanned vehicles.

We argue here that this simple idea offers great potential in helping to realise the vision of high performance ITSs, in particular, in realising systems in which high precision vehicle localisation is used to underpin a wide variety of location-based services. We make this claim for a number of reasons. First, an average vehicle remains parked for long periods, during which it might collect large sets of GNSS data from its static location to highly improve the accuracy of its position estimation by e.g. using post-processing tools (7). Thus, parked cars might go from being unused pieces of specialised/expensive hardware, to becoming a network of reliable reference points for the localisation process of nearby vehicles. Second, in most cities, parked cars offer a dense and flexible network, with a wide geographical distribution; thus, they can be used as a cheap option to augment (or even supplant) RSUs for applications requiring densely deployed infrastructure such as positioning/routing of autonomous vehicles $(2 ; 3)$. Moreover, also due to dense deployment, the infrastructure is potentially robust, meaning that not all parked cars need to participate (a selection algorithm can filter out the optimal subset of participating vehicles), and some units can even fail without affecting the network fidelity (broken nodes can be rapidly overtaken by unselected ones). Finally, as the vehicle fleet refreshes itself over short time-scales, replacement and maintenance of infrastructure is considerably simpler than in the case of dedicated RSUs.

This simple idea also follows recent works that have recognised the ability of suitably equipped stationary vehicles as general service delivery platforms $(8 ; 9)$. Our purpose here is to show that such a novel idea has the potential of greatly improving current CP systems, and in turn position- 
based control systems (e.g., waypoint tracking), without requiring any dedicated infrastructure, and at the same time opening new markets for vehicle owners, cities and municipalities, as well as car manufacturers to monetise vehicles in a non-traditional manner. Our vision imagines that vehicle owners would make their vehicles available for such applications in exchange for monetary compensation, or for access to privileged services and facilities such as parking spots.

This paper is organised as follows. In Section 2, we examine the related work concerning the use of stationary vehicles in providing VANET-related services. In Section 3, the main problem of interest, together with the main definitions and assumptions used in our work are given. In Section 4, we provide a discussion about some supporting facts to assess the feasibility of the use of stationary powered-on and powered-off vehicles in ITS applications in general, and in CP tasks in particular. Section 5 is devoted to describe how stationary vehicles can be embedded in existing CP algorithms to improve the results. Experimental evaluations and a non-traditional application case are presented in Section 6. Finally, Section 7 concludes the paper and outlines current and future lines of ongoing research in the same direction.

\section{Related work: Stationary vehicles supporting the delivery of VANET-related services}

$\mathrm{CP}$ is a topic of high interest in many communities, with a large range of positioning techniques reported in the specialised literature $(4 ; 5 ; 10)$. In the particular case of VANETs, the improvements in the performance of $\mathrm{CP}$ systems are generally analysed in terms of new developments on ranging/rating techniques $(5 ; 11 ; 12)$, sensing/communication technologies $(5)$, localisation algorithms $(13 ; 14)$, and properties of the measurement noise $(15)$. However, there is a less-explored alternative for the same purpose: the enhancement of contextual conditions for deploying CP systems, which has a direct impact on the accuracy provided by the CP algorithms. The above is supported in the basic premise that if more/better sources of contextual information are available, then more elaborated results (i.e. position estimations) can be obtained.

In this vein, stationary vehicles (those with time-invariant position) have recently received special attention as pivotal elements to enlarge the sources of reliable contextual data, such as the availability of nearby free parking spaces or the detection of a special "passing-by" device (8), and to support the provision of dedicated services such as content downloading and distribution $(16 ; 17)$, improvement of multi-channel operations $(18 ; 19)$, mitigation of signal attenuation in ITS applications $(20)$, and their potentiality as RSUs $(21 ; 22)$, all of this thanks to the advent of modern ITS technologies allowing Vehicle-to-Vehicle/Infrastructure (V2X) communication and cooperative awareness.

As can be noticed, the use of parked vehicles for CP applications is still an unexplored field of research, and the objective of this paper is to provide a preliminary assessment of its potential.

\section{Problem definition and assumptions}

\subsection{Problem definition}

The objective of a localisation process is to obtain an accurate estimation of the absolute position of a vehicle (e.g., in terms of its latitude and longitude coordinates). In a CP process, we shall denote by blind vehicles the vehicles whose position is not known, by target vehicle a specific blind vehicle that becomes interested in knowing its position, and by neighbouring vehicles some vehicles that are within the communication range (i.e. data exchange area) of the target vehicle. Typically in a $\mathrm{CP}$ process, neighbouring vehicles will support the target vehicle in estimating its position in an accurate and quick manner. In addition, we shall denote by stationary vehicle a vehicle 
that remains static longer than two consecutive sampling instants $(2$ seconds in our simulated experiments). Accordingly, stationary vehicles can remain static for a few seconds or minutes in the case of powered-on stationary vehicles, such as those that may be stopped at traffic-light or in bottlenecks, or for hours, as in the case of powered-off stationary vehicles, such as parked cars.

\subsection{Data and general assumptions}

\subsubsection{Parked vehicles' information for experimental evaluation}

We use publicly available data to harvest the density of parked vehicles in typical urban scenarios. For this, Earth/street views from Google Maps are used to manually identify parked vehicles, of which their relative positions were mapped to the coordinate system of the simulation environment to generate a benchmark of simulated parked vehicles.

Remark 1: Latitude-longitude coordinates from Google Maps were never used in our simulated experiments as these are typically unreliable; instead, we used image processing, scaling, rotations and translations to map the relative positions of identified parked cars into the simulation environment.

\subsubsection{Assumptions and facts concerning vehicles}

In our simulated experiments, each vehicle is assumed to have:

- a GPS logger, to measure and register detailed GPS information, including latitude-longitude coordinates and the associated Horizontal Dilution-Of-Precision (HDOP) information;

- a V2V communication system, to perform V2V ranging and exchange relevant vehicle's information including speed and state of the vehicle (powered-on, powered-off), among others;

- an on-board unit (OBU), to store and process the collected data.

Concerning GPS signals, simulated positioning error is assumed to be a zero-mean Gaussian variable with standard deviation (S.D.) $\sigma_{G P S}=6 \mathrm{~m}$, consistent with $90 \%$ of the positioning errors less than $10 \mathrm{~m}$. Some of our simulations are supported in real-world GPS measurements, for which we use two different kinds of GPS receivers: the smartphone Samsung Galaxy SIII mini with the GPSLogger ${ }^{1}$ app, and the travel recorder Qstarz BT-Q1000TX, hereafter referred as SIII and Q1000TX, respectively.

Concerning the V2V communication system, we assume that the communication range is of 15 $\mathrm{m}$, corresponding to Class-A Data Short-Range Communication (DSRC) devices (23), where the ranging error is assumed to be a zero-mean Gaussian variable with S.D. $\sigma_{R} \in\{0.2 \mathrm{~m}, 4 \mathrm{~m}\}$, consistent with the values used in $(24 ; 25)$, where $\sigma_{R}=0.2 \mathrm{~m}$ corresponds to Ultra-Wide Band (UWB) based ranging and $\sigma_{R}=4 \mathrm{~m}$ corresponds to Radio Signal Strength (RSS) based ranging. Note that we do not emulate the V2V process itself; rather, communications errors arising from the transmission process and protocol design are dealt with in a lumped manner using noise parameters.

Remark 2: Accuracy levels of less than $3.5 \mathrm{~m}$ have been reported for high-quality GPS receivers at a given surveyed benchmark (26). Our choice of $\sigma_{G P S}=6 \mathrm{~m}$ follows experimental results using commercial receivers in real-world settings (27).

Remark 3: In this study we do not explicitly consider multipath effects and possible shadowing consequences in the context of V2V ranging, as we assume a direct Line-Of-Sight (LOS) between the target vehicle and its neighbours as a consequence of the used communication range (i.e. 15

${ }^{1}$ https://play.google.com/store/apps/details?id=com.mendhak.gpslogger 
$\mathrm{m})$. Under this assumption, such effects are mitigated (25; 28). Accordingly, in the remainder of the paper we shall only consider Class-A DSRC devices- the underlying assumption being direct LOS.

\section{Feasibility of the idea of using stationary vehicles for CP purposes}

We now give some real-world facts and numbers to support the feasibility and further assess the potential of using stationary vehicles for CP applications.

1) Battery issues: Currently, two types of stationary vehicles can be distinguished as active sources/pivots of contextual information: (i) powered-on vehicles, such as vehicles temporarily stopped in a queue (e.g., (19)); and (ii) powered-off vehicles, such as parked vehicles (e.g., (20)). While the usage of powered-on stationary vehicles does not pose any particular technical requirement in the provision of new services, an obvious concern associated with parked cars (as the main class of powered-on stationary vehicles) is battery discharge. This concern is investigated in (29), where the authors present a study about the impact of the communication system and data processing unit on the level of charge of a parked car's battery; the main conclusion is that the power demanded from the analysed on-board components is not particularly significant: given a fully charged battery, services can be provided for up to few days before a critical point of the charge level is reached. In fact, the main technical challenge is keeping the on-board communication systems switched on when the vehicle is powered off. In particular, by accessing a maximum of $10 \%$ of the charge of a 480 Wh car's battery, it has been recently demonstrated that a $1 \mathrm{~W}$ on-board unit can be constantly powered for up to 2 days (20).

2) Availability of stationary powered-on vehicles: The average time spent in stop/idle mode between two consecutive intersections in urban scenarios can reach up to $50 \%$ of the whole time spent along the arterial road (30). Idle times become even larger at pm rush hours as people drive in a more relaxed way since they feel that they do not have strong time constraints in returning home, which causes longer queues at signalised/controlled intersections (31).

Accordingly, a large number of vehicles stuck in such queues may possibly be used with high priority for positioning applications. To experimentally support this, we estimate the portion of the area covered by vehicles stuck in heavy traffic in Dublin's City Centre, Ireland (Fig. 1) with respect to the overall analysed network, using AA Roadwatch as data source (a service offered by AA Ireland ${ }^{2}$ ). Data were collected between 16th-23rd June, 2014 at lunchtime/evening rush hours (1 pm and 7:30 pm, respectively). The analysis of the collected data is reported in Table 1 , from which it is possible to conclude that (in the context of this small survey) an average of $22.64 \%$ and $15.71 \%$ of the main streets in Dublin's City Centre are occupied by stationary powered-on vehicles at the rush hours of working days. Even though numbers decrease at the weekends (14.36\% at lunchtime and $13.03 \%$ in the evening), they still represent a significant coverage of the total network analysed, specially around intersections (as shown with black curves in Fig. 1.b).

Table 1. Sample of congested traffic over total traffic at different rush hours for the main streets in Dublin's City Center.

\begin{tabular}{|c|c|c|c|c|c|c|c|}
\hline Rush hour & MON & TUE & WED & THU & FRI & SAT & SUN \\
\hline Lunchtime & $18.39 \%$ & $17.68 \%$ & $25.03 \%$ & $23.49 \%$ & $28.63 \%$ & $14.95 \%$ & $13.77 \%$ \\
\hline Evening & $13.07 \%$ & $16.17 \%$ & $14.58 \%$ & $20.67 \%$ & $14.07 \%$ & $9.72 \%$ & $16.33 \%$ \\
\hline
\end{tabular}

3) Availability of stationary powered-off vehicles: A car is typically parked on average up to 23 hours a day $(32 ; 33)$, and usually outdoor $(16 ; 20)$. For example, in a recent study of

${ }^{2}$ http://www.theaa.ie/AA/AA-Roadwatch.aspx 


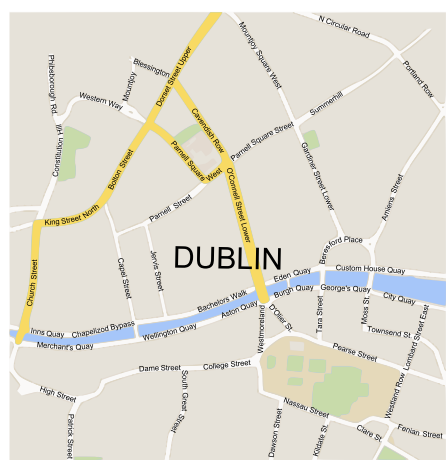

(a) Selected area.

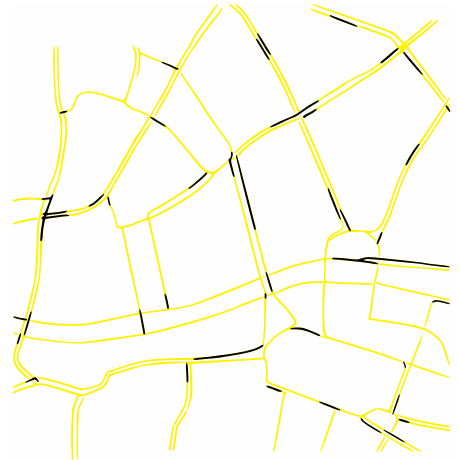

(b) Example of traffic data.

Figure 1. Roads in Dublin's City Centre monitored at lunchtime and evening rush hours. Color convention for monitored roads: black sections, occupied by stationary powered-one vehicles; yellow sections: free traffic.

61,000 daily parking events in Montreal City (34), 69.2\% of all parked cars were parked on-street and $27.1 \%$ were parked in outside parking lots, while only $3.7 \%$ were parked in interior parking facilities, with an average duration of on-street parking of 6.64 hours. Further, current predictions concerning regulated parking spaces claim that the average portion of on-street parking spaces, as a percentage of the overall total number of parking spaces, will be $30.17 \%$ (with up to $56.22 \%$ for Italy and $43.30 \%$ in Spain) (35).

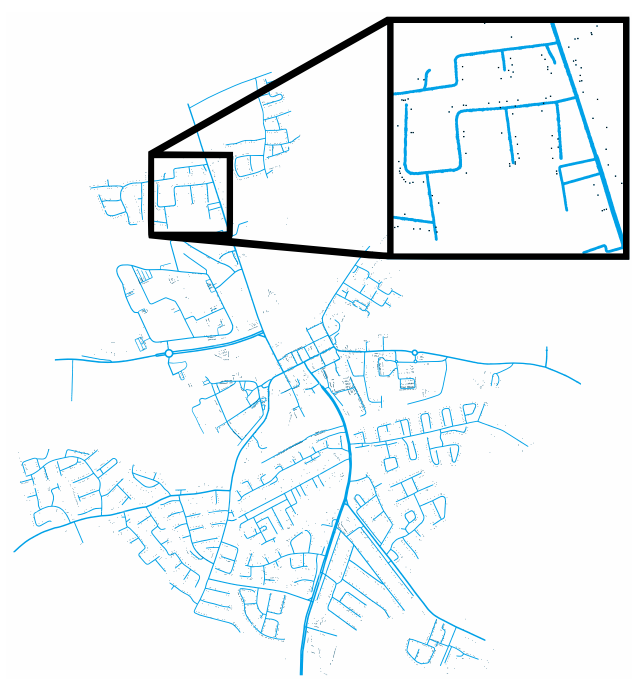

(a) Roads (blue) and parked cars (black).

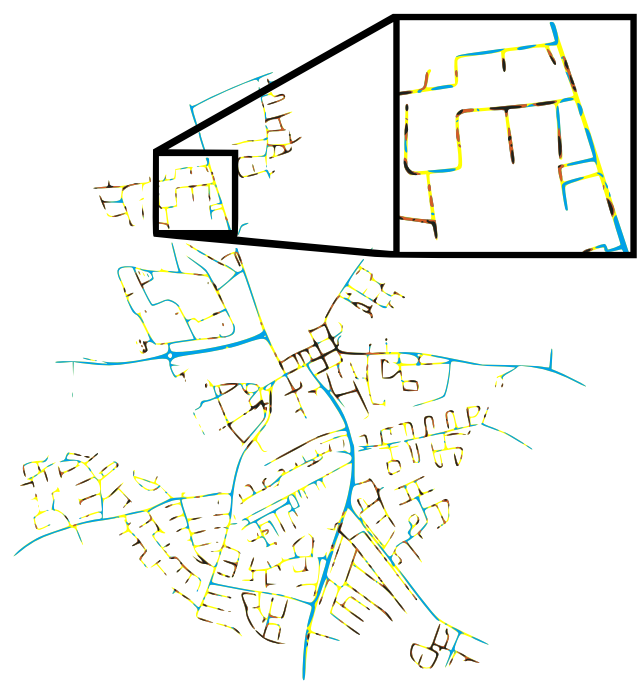

(b) Covered areas using a Class-A DSRC devices.

Figure 2. Maynooth, Co. Kildare, Dublin. A total of 4541 vehicles were identified as parked vehicles using satellite imagery from Google Maps. Color convention for levels of coverage: yellow, covered by 1 parked vehicle; orange, covered by 2 parked vehicles; red, covered by 3 or more parked vehicles.

Thus, parked cars can be thought of as an unused dense network of dedicated sensors with no strong power/collection constraints, and whose position can eventually be known with high accuracy to help CP problem for ITS systems, acting like a flexible network of RSUs. To experimentally support this, we estimate the portion of the area for vehicular transit that can be covered by parked cars in function of a realistic geographical distribution and a communication zone of 15 m (DSRC devices class A). For this, we analyse the urban area of Maynooth, Co. Kildare, Ireland, with parked cars identified following Section 3.2.1 (Fig. 2.a). The resulting area coverage is shown 
in Fig. 2.b, equivalent to $27.78 \%$ covered by 1 parked vehicle, $11.98 \%$ covered by 2 parked vehicles, $14.81 \%$ covered by 3 or more parked vehicle, with $45.43 \%$ of uncovered area. Thus, $54.57 \%$ of the area for vehicular transit can be covered at least by one car using Class-A DSRC devices, which can be seen as the lower bound of coverage with respect to other classes of DSRC devices (23).

Finally, note that the total area coverage becomes greater once parked vehicles and stationary powered-on vehicles are simultaneously considered: stationary powered-on vehicles mainly contribute with coverage around intersections, while on-street parked vehicles are usually densely distributed along the roads.

\section{Methodology}

As we have already mentioned in Section 2, our objective is not to develop a novel CP algorithm, but to show how stationary vehicles can be embedded in existing CP algorithms to improve their efficiency and their accuracy. For convenience, we now briefly recall two popular CP algorithms, and explain how to proceed to include stationary vehicles in the CP process.

\subsection{Range-based CP algorithms}

Among the variety of range-based CP algorithms reported in the literature (e.g., $(4 ; 5 ; 11)$ ), we evaluate the performance of two CP algorithms as reference exemplars. The first CP algorithm chosen is a decentralised estimation algorithm based on Guaranteed Convergence Particle Swarm Optimiser (GCPSO) (36), using inter-vehicle distance measurements to minimise the cost function

$$
f\left(\hat{\mathbf{x}}_{i, t}\right)=\sum_{\forall j \in S}\left\|d_{i j, t}-\right\| \hat{\mathbf{x}}_{i, t}-\mathbf{x}_{j, t}\|\|^{2}+\left\|\hat{\mathbf{x}}_{i, t}-T_{s} \mathbf{v}_{i, t-1}\right\|^{2},
$$

where $i$ is the index of the target vehicle, $\hat{\mathbf{x}}_{i, t}$ is the estimate of the real (unavailable) 2D position $\mathbf{x}_{i}$ of target vehicle at time step $t, S$ is the set of neighbours of the target vehicle, $\mathbf{x}_{j}$ is the available $2 \mathrm{D}$ position of neighbouring vehicle $j$ shared to the target vehicle, $\mathbf{v}_{i}$ is the $2 \mathrm{D}$ speed of the target vehicle, $T_{s}$ is the updating time, and $d_{i j}$ is the measured distance between the target vehicle and the neighbour $j$. Thus, this is a distributed, range-based CP algorithm based on a heuristic optimiser.

The second CP algorithm chosen is a simplistic version of the Extended Kalman Filter (EKF) (24) which fuses inter-vehicle distance measurements and vehicle kinematics (speed information), to obtain a sequential Bayesian estimation of a target vehicle's position. Thus, this is a distributed, range-based, Bayesian CP algorithm. For a detailed description of this algorithm please refer to $(24)$.

Finally, as in 2D position estimation a minimum of 3 inter-vehicle distances are required for a target vehicle to narrow down its possible location (4), we set this number as the upper bound of allowed participating neighbours in the $\mathrm{CP}$ process of a target vehicle merely for practical reasons (a real-world implementation can include as many neighbours as desired), where the "best" subset of 3 neighbours is chosen through the selection strategy described below. Ultimately, if no neighbours are available, position estimations are obtained using dead reckoning from speed measurements fused with GPS data via Kalman filtering (see Sections 3.2.2 and 6 for the noise properties to use).

\subsection{Node-selection strategy to enhance CP algorithms}

A smart selection of the neighbouring nodes for CP can speed up the localisation procedure, mitigate energy requirements and, consequently, reduce battery consumption; an example of this is presented 
in (37), where an optimal subset of anchor nodes is chosen based on their geometric dilution-ofprecision. We now show how stationary vehicles can be conveniently included in the CP algorithm; for this purpose, we adopt a simple deterministic selection strategy based on priorities of neighbours (broadcasted through their $\mathrm{V} 2 \mathrm{~V}$ communication systems), according to the following reasoning.

A target vehicle may have access to 2 types of neighbours for CP purposes: stationary and moving vehicles. Generally speaking, the position information is more accurate for the former than for the latter, which is simply motivated by the more dynamic nature of moving vehicles. From this, we propose to rank the participating vehicles as shown in Table 2.

Table 2. Priorities for participating vehicles in the CP process of blind cars (P1 is the highest priority).

\begin{tabular}{|c|c|c|}
\hline Priority & Main condition & Extra condition \\
\hline \hline P1 & Parked car. & Estimated location with acceptable level of dispersion. \\
\hline P2 & Powered-on stationary car. & At least 3 neighbours with priority in $\{$ P1,P2 $\}$. \\
\hline P3 & Moving car. & At least 3 neighbours with priority in $\{$ P1,P2 $\}$. \\
\hline P4 & Any other case. & Any other case. \\
\hline
\end{tabular}

The main reason for giving a higher priority to parked cars resides mainly in their expected period of stationarity (up to hours), that generally corresponds to a more accurate knowledge of their own position. Additionally to the accuracy of the localisation procedure, another important parameter is the level of dispersion of the estimated position of a parked car, which we shall compute as

$$
\epsilon(t)=\sqrt{S_{\mathrm{lat}}(t)^{2}+S_{\mathrm{lon}}(t)^{2}}
$$

where $S_{\text {lat }}(t)$ and $S_{\text {lon }}(t)$ are the sample S.D. (in meters) of the vectors of latitude-longitude estimates in a given period $[t-\tau, t], t>\tau$, respectively. Thus, $\epsilon$ provides an indication of how dispersed the position estimates are with respect to the sample mean in the same period; in particular, note that about $95 \%$ of such estimates lie within a range of at most $2 \epsilon$ meters. In the following, we shall take $\epsilon_{\max }=0.5$ as an upper bound of $\epsilon$ to presume that the positioning process has converged to a steady-state value, as $\epsilon$ smaller than 0.5 corresponds to a variation smaller than $1 \mathrm{~m}$ (on average) in $[t-\tau, t]$. Finally, the selection strategy consists in choosing the 3 neighbours with the highest priorities; given the availability of neighbours with equal priority, we then choose the closest to the target car. Ultimately, if fewer than 3 are available, we then choose the highest available number.

\subsection{Localisation of parked vehicles}

We estimate the position of parked cars using averaged GPS data (equivalent to basic Kalman Filtering) which is more accurate than using instantaneous measurements, enhanced by CP with averaged ranging data from other parked cars, if available. In particular, we shall average a collection $\left\{\left(\operatorname{lat}_{i}, \operatorname{lon}_{i}, \mathrm{HDOP}_{i}\right)\right\}_{i=1, \ldots, N}$ of $N$ GPS measurements using: i) Standard Average (SAVG) of the GPS measurements with HDOP $i \leq 5$, and ii) Weighted Average (WAVG) of all GPS measurements, with weighting factors $\lambda_{i}$ given by the Mean Inverse Square of the HDOPs, calculated as

$$
\lambda_{i}=\frac{\frac{1}{H D O P_{i}^{2}}}{\sum_{j=1}^{N} \frac{1}{H D O P_{j}^{2}}} .
$$

\subsection{General procedure to localise blind vehicles}

Finally, we adopt the following iterative procedure to localise blind vehicles: 
(1) Detect available neighbours and collect the relevant information from them.

(2) Estimate the location of the target vehicle:

- if target vehicle is not parked: use a positioning algorithm (e.g. from Section 5.1) combined with the node-selection strategy in Section 5.2.

- if target vehicle is parked: use the localisation approach in Section 5.3.

(3) Update the parameters of the target vehicle: priority (according to Table 2), and level of dispersion (according to Eq. (1)) if parked.

\section{Performance evaluation}

To evaluate the performance of the proposed approach we use simulated experiments, some of which are supported by real-world GPS data. In addition, we use the following parameter settings:

- general setup:

o updating time $T_{s}$ for the localisation algorithms in simulated experiments: $1 \mathrm{~s}$.

- parameters for the GCPSO algorithm given in (36):

○ 4 particles, 50 iterations, $s_{c}=15, f_{c}=5, \rho=1, c_{1,2}=2$, linearly decreasing inertia weight from 0.9 to 0.2 , minimum fitness value for stopping criteria $=0$, initial swarm: 2 particles at best neighbour's position and 2 particles at $T_{s} * \mathbf{v}_{i, t-1}$;

- parameters for the EKF algorithm given in (24):

$\circ \mathbf{Q}_{k-1}=\sigma_{\mathbf{Q}}^{2} \mathbf{I}, R_{k}=\sigma_{R}^{2} \mathbf{I}, \boldsymbol{\Gamma}_{k-1}=\sigma_{\boldsymbol{\Gamma}}^{2} \mathbf{I}$, with $\mathbf{I}$ the identity matrix with appropriate dimensions, and with $\sigma_{\boldsymbol{\Gamma}}=0.5, \sigma_{\mathbf{Q}}=2$ and $\sigma_{R}$ as in Section 3 .

\subsection{Levels of accuracy for the localisation of parked vehicles}

This subsection is devoted to evaluate the potential accuracy of the position estimations for parked vehicles, through the use of field data merged with simulated ranging values, within a simulation environment built in Matlab. For this, we use real-world GPS data collected from three parking spots in UCD Campus, Belfield, Dublin, Ireland, where some relevant surrounding obstructions are present (buildings and trees). As no geodetic marks were available for those three locations, WAVG of GPS measurements from the Q1000XT during 3 hours, with corrections from the real inter-vehicle distances (measured by hand), were used to estimate the ground truth references, obtaining: Location 1, (53.307144, -6.217872); Location 2, (53.307206, -6.217827); Location 3, (53.307111, -6.217686).

In the numerical evaluation, we applied the positioning approaches in Section 5.3 with the GCPSO-based CP algorithm from Section 5.1, $\sigma_{R}=0.2 \mathrm{~m}$ for the simulated V2V ranging, and real-world GPS data collected every 10 seconds for 90 minutes through a SIII GPS receiver at each location. The results of the experiment are presented in Fig. 3.

Recall the positioning errors shown in Fig. 3 are with respect to the estimated ground truth references, and thus this experiment basically provides the evolution of the position estimation using "cheap" GPS receivers (i.e. those from the phones) with respect to the best position obtained from a dedicated GPS receiver (the Q1000TX) and post-processing tools. Two important conclusions can be drawn from this experiment. First, results show a convergence of the average absolute positioning error below $3 \mathrm{~m}$ in just a period of $24 \mathrm{~min}$, with slightly better performance for the WAVG-based $\mathrm{CP}$ approach, and below $1.47 \mathrm{~m}$ in a period of $90 \mathrm{~min}$, this last relevant enough with respect to the average dimensions of a mid-size sedan (about $2 \mathrm{~m}$ wide and $4.5 \mathrm{~m}$ long). And second, we can see that both SAVG-based and WAVG-based position estimations can be considered reliable enough (in terms of the chosen $\epsilon_{\max }=0.5$ and $\tau=30 \mathrm{~min}$ ) from $t=52 \mathrm{~min}$ for location 1 , from $t=61 \mathrm{~min}$ for location 2, and from $t=54 \mathrm{~min}$ for location 3, and thus the analysed parked cars can obtain priority P1 after the corresponding times. Note that the longest of these times represent only a $15.3 \%$ of the average on-street parking time reported in (34), which supports our assumption that 

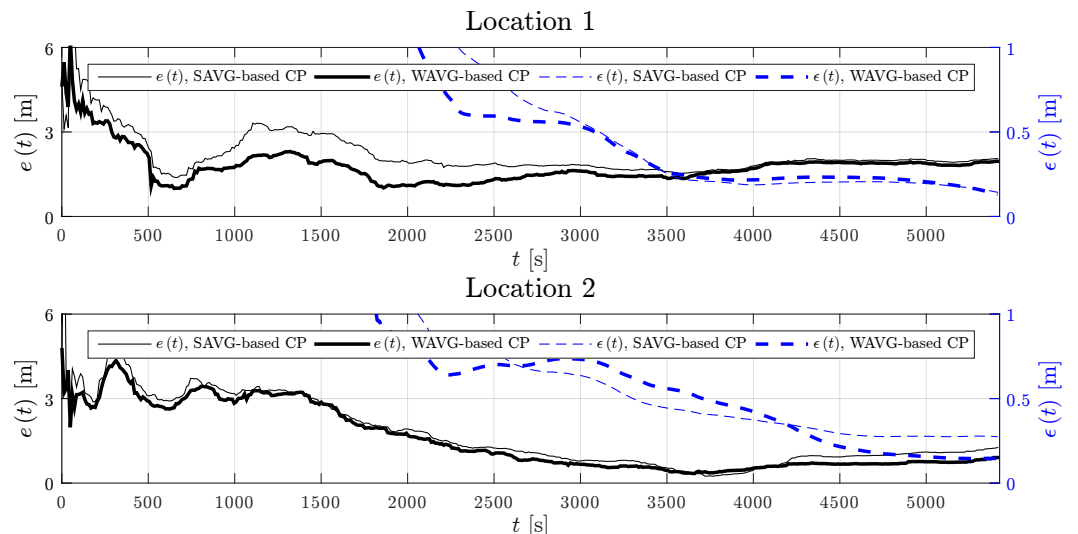

Location 3

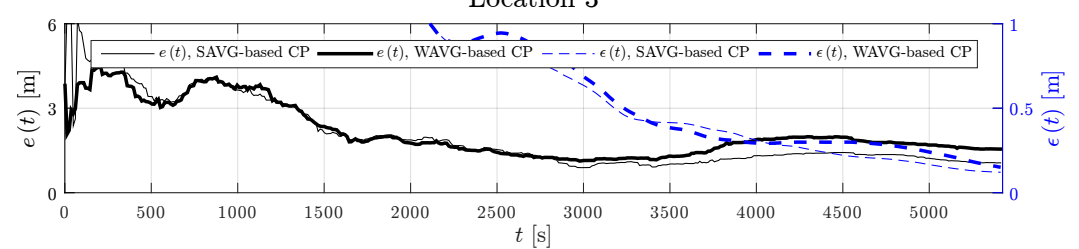

Figure 3. Results of two localisation approaches using real-world GPS data from 3 locations in UCD Campus: absolute positioning error $e(t)$, and level of dispersion of the estimations $\epsilon(t)$ (Eq. 1) with $\tau=30 \mathrm{~min}$. Initial values of $e(t)$ are caused by cold/normal start of the GPS receivers.

parked cars do not have strong time constraints (on average) for achieving accurate localisation. In addition, by replacing SAVG/WAVG with a more elaborated autonomous estimation method as the one reported in (38), all the above numbers can be greatly improved, although by sacrificing the low computational complexity of the averaging methods.

Accordingly, in the following simulations we shall use the outcomes of this experiment to set the statistical parameters of the positioning error of parked cars. In particular, we will assume that such a positioning error can be modeled as a normal random variable $N\left(0, \sigma_{P}\right)$ with $\sigma_{P}=2 \mathrm{~m}$, this corresponding to about a $70 \%$ of the parked cars localised with an accuracy of less than $2 \mathrm{~m}$.

\subsection{Localisation of blind vehicles in a large-scale scenario}

We now evaluate our proposed CP approach using a simulation environment built with the SUMO $^{3}$ simulator, a free and open traffic simulation suit commonly used in the ITS community, and using digital maps imported from OpenStreetMap ${ }^{4}$, a community-driven digital map provider. In addition, Python scripts are used to online interact with SUMO simulations (e.g., to perform the localisation process of blind vehicles).

The investigated large-scale scenario consists of the road network of main secondary and tertiary roads from Maynooth Town, Ireland, and 605 parked cars located in a proximity of $15 \mathrm{~m}$ to the selected roads (identified according to Section 3.2.1), as shown in Fig. 4. In addition, we evaluate two scenarios for the localisation error for parked cars: the ideal case, i.e. where the position of parked vehicles is assumed to be known exactly, and the more realistic case where we simulate the uncertainty about the exact position of the parked vehicles by corrupting their actual position with some noise. Thus, we assume that all the parked vehicles are immediately available from the beginning of the simulated experiments with priority $\mathrm{P} 1$, and with positioning errors in $N\left(0, \sigma_{P}\right)$ and $\sigma_{P} \in\{0 \mathrm{~m}, 2 \mathrm{~m}\}$. Note that as discussed in Section 6.1, this corresponds to investigating the accuracy of the $\mathrm{CP}$ algorithms under the assumption that the position of parked vehicles is known

\footnotetext{
3 www.dlr.de/ts/sumo/en/

$4_{\text {www. OpenStreetMap. org }}$
} 
perfectly $\left(\sigma_{P}=0\right)$, or taking into account possible localisation errors of the GPS devices.

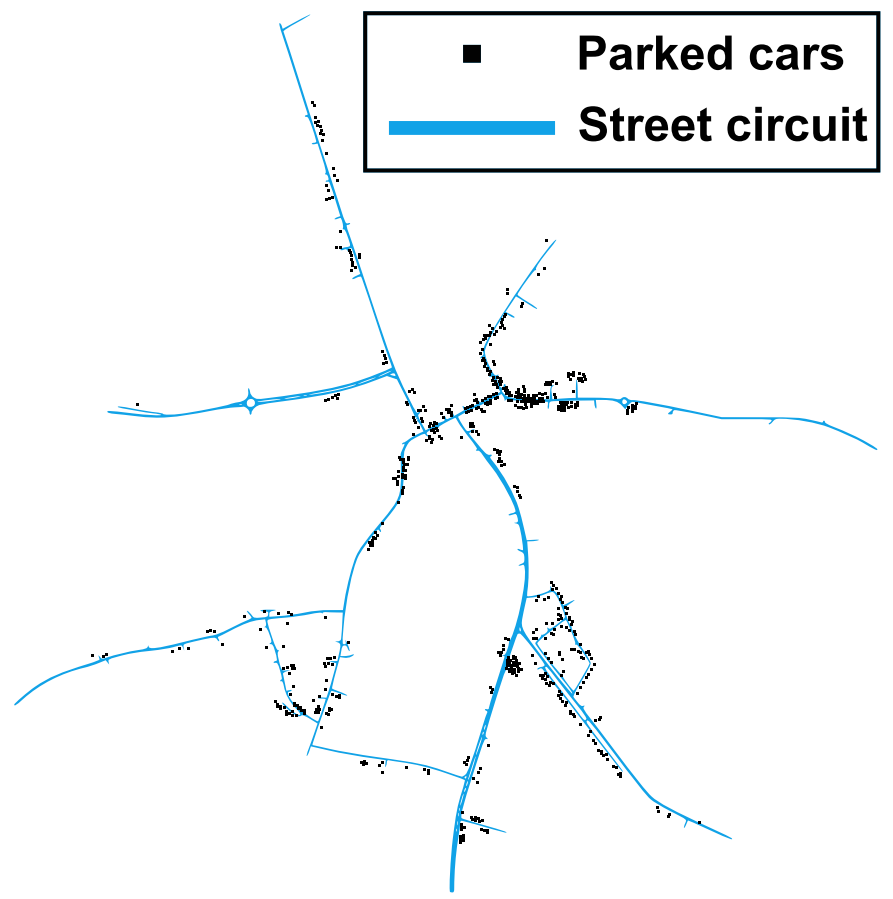

Figure 4. Large-scale localisation test: street circuit (blue), and 605 selected parked cars (black).

Our simulated experiments are constructed as follows: we assume that 200 vehicles are in the road network at the beginning of the simulation, and then new vehicles enter the network at a rate of one every $4 \mathrm{~s}$. Routes for the participating cars are randomly chosen, but recorded to be used in the repetitions of the simulated experiments with a different combination of the experimental setup, in terms of the accuracy of the localisation of parked cars (i.e. $\sigma_{P} \in\{0 \mathrm{~m}, 2 \mathrm{~m}\}$ ), the accuracy of the communication system (i.e. $\sigma_{R} \in\{0.2 \mathrm{~m}, 4 \mathrm{~m}\}$ where we remind that $\sigma_{R}=0.2$ implies the use of UWB-based ranging techniques, while less accurate results are obtained when RSS-based ranging techniques are used instead, i.e., $\sigma_{R}=4$ ) and the use of an underlying different CP algorithm (i.e., GCPSO and EKF). Finally, each experiment is repeated 10 times, to average out the stochastic effects.

From the simulated experiments, 245 of the overall 257 cars in the network encountered at least 1 parked car along their routes. Average values of the Mean Absolute positioning Error (MAE) for the entire set of cars are shown in Fig. 5, from which a reduction of the MAE is evident when the proposed approach is used. In particular, it is important to note that similar improvements are obtained in all 8 cases, i.e., whatever is the specific CP algorithm employed, whatever is the communication technology, and independently on whether the position of parked vehicles is assumed to be known exactly or not. Such a result confirms that the use of stationary vehicles does actually improve the accuracy of traditional cooperative positioning systems, independently from the specific underlying software (i.e., algorithm) or hardware (i.e., employed devices) used by the traditional method. A more detailed analysis is provided in Fig. 6. Here, the improvement of the localisation accuracy is shown together with the number of parked vehicles encountered during its trajectory. From this figure, it is possible to appreciate that in all the 8 analysed cases, the biggest improvements are obtained when more parked vehicles are encountered, while obviously there are no (or negligible) improvements when a vehicle does not encounter parked vehicles along its route (or only meets a few of them).

While figures 5 and 6 show that in general the information provided by the parked vehicles improves the localisation accuracy in general, still, as one might largely expect, it is possible to 

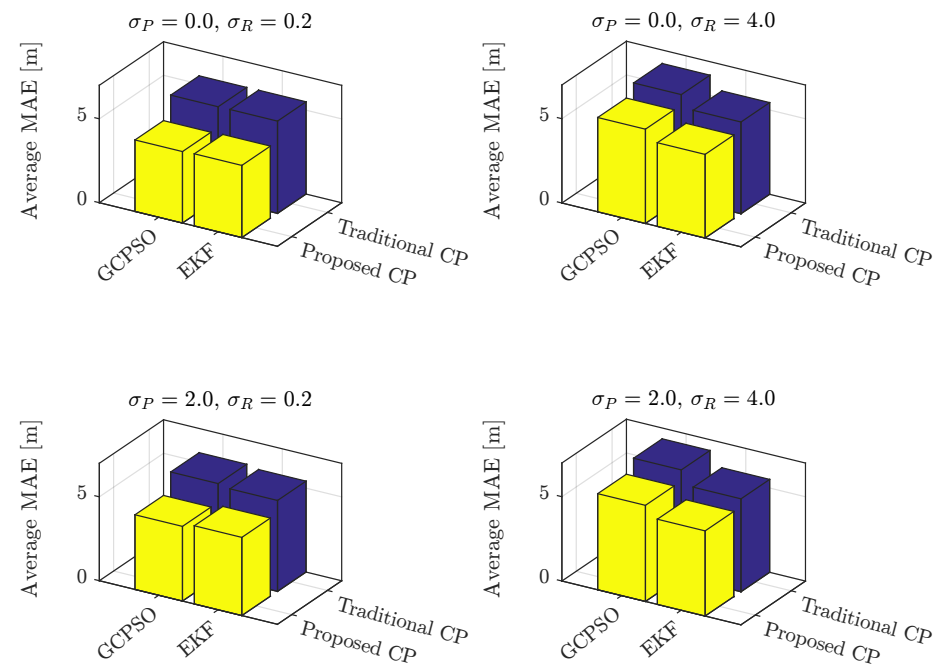

Figure 5. Numerical results for the large-scale localisation test: Average value of the MAEs for the set of 257 target cars. A reduction of the MAEs is observed for all the subplots when using the proposed CP approach.
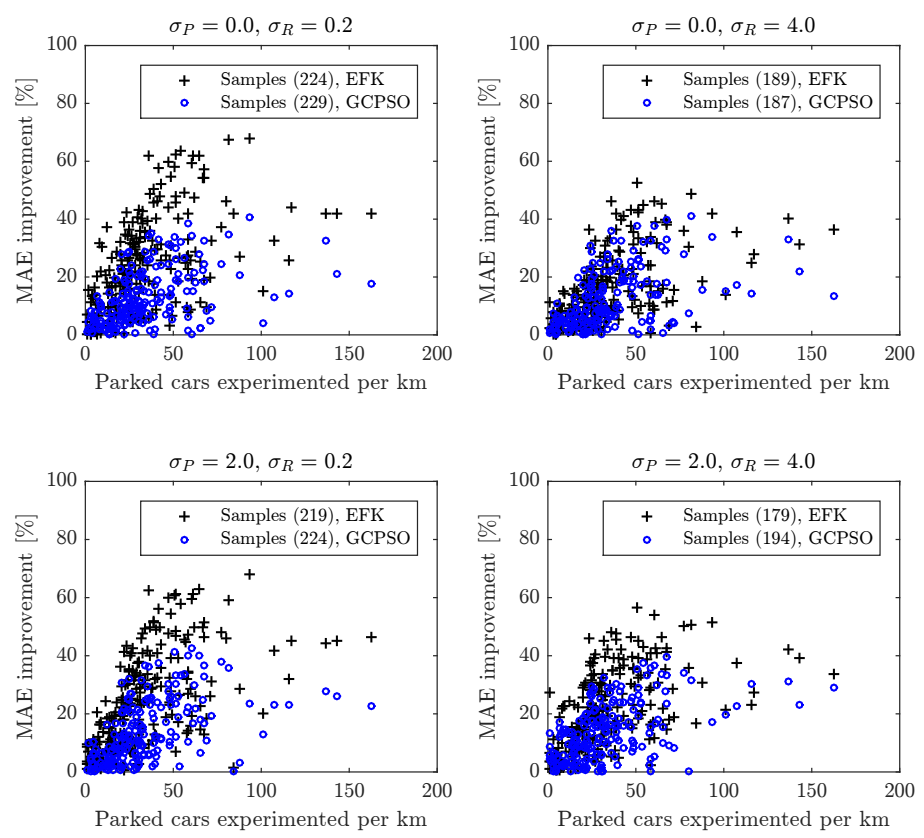

Figure 6. Numerical results for the large-scale localisation test: Improvements of the MAEs (Proposed approach over Traditional approach) versus the number of parked cars experimented per $\mathrm{km}$ by different target cars. Each sample corresponds to a target car experimenting at least 1 parked car and with positive MAE improvement. [Total number of target cars: 257, of which only 245 experimented at least 1 parked car.]

note that the largest improvements can be achieved when the position of parked vehicles is known perfectly (rather than with some localisation error) and when a UWB-based communication system is adopted. Finally, Fig. 7 compares all the estimated trajectories (i.e., within the Monte Carlo ensemble) with the actual trajectory for a specific vehicle. From this figure it is possible to appreciate 
that the estimate is very accurate when more parked vehicles are encountered, in the middle of the trajectory, rather than at the beginning and at the end of the trajectory when fewer or none vehicles are parked.
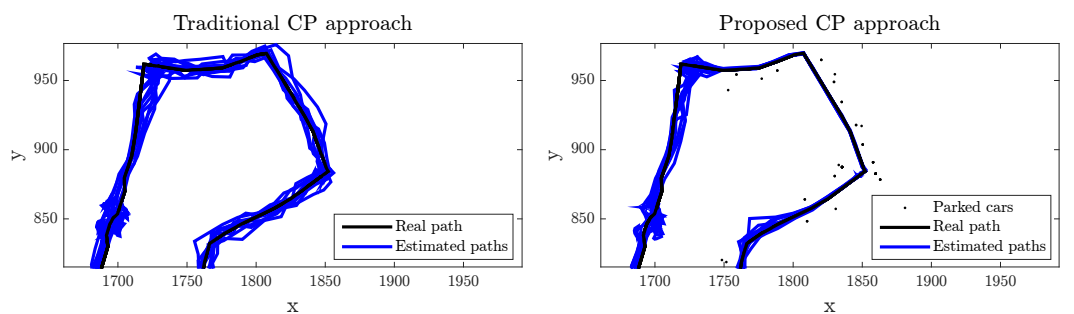

Figure 7. Numerical results for the large-scale localisation test: example of performance improvement in the positioning estimation process of a target car when using the proposed approach. [Set-up used: GCPSO, $\sigma_{P}=0 \mathrm{~m}$ and $\sigma_{R}=0.2 \mathrm{~m}$ ].

\subsection{Application case: mobility of unmanned vehicles}

Now we evaluate an application case beyond the context of traditional vehicular mobility: the waypoint tracking of a drone. In this application case, our objective is to use parked vehicles to support rapid drone navigation in urban scenarios. In our simulated experiments, we use the simulation model and the waypoint tracking approach from the AR Drone Simulink DevelopmentKit V1.1 ${ }^{5}$ (39) for use with Maltab. The desired path is made of the road segments Brendan Rd, Arranmore Rd, Herbert Park Rd and Pembroke Park Rd in Dublin, Ireland (i.e. an urban environment), and only consider parked cars for practical reasons, as shown in Fig. 8, where the starting point is the extreme of the path at the bottom of the figures. Using Google imagery and the procedure described in Section 3.2.1, 129 parked cars were identified for this path. In addition, a proper communication protocol between the on-board devices carried by the drone and the parked cars was considered (e.g., by using a mobile DSRC equipment ${ }^{6}$ ) with a communication range of $100 \mathrm{~m}$ and $\sigma_{R}=0.2 \mathrm{~m}$. Here, the high used communication range (equivalent to a DSRC device Class B (23)) was motivated by the fact that the LOS between the drone and the parked cars is potentially less blocked than the LOS between a land vehicle and parked cars (the drone moves at a higher level than the one of the parked cars).
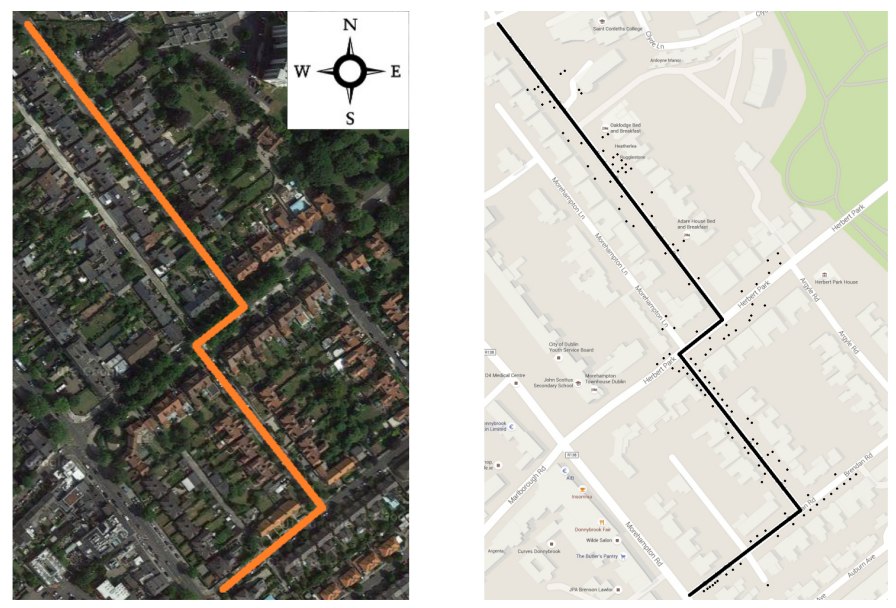

Figure 8. Common scenario for the non-traditional application cases: left, top-view picture from Google maps of the proposed path to be followed; right: relative position of identified parked cars (black dots) from Google imagery (top-view and street-view).

${ }^{5}$ http://www.mathworks.com/matlabcentral/fileexchange/43719-ar-drone-simulink-development-kit-v1-1

${ }^{6} \mathrm{http}: / /$ www.aradasystems.com/locomate-me-as-the-mobile-dsrc-device-naias/ 
A performance index to be analysed is the discrete integral of the tracking error, defined as

$$
I_{T}=\sum_{k=1}^{N}\left\|p_{\text {pos }}^{(k)}-w_{\text {pos }}^{(k)}\right\|_{2},
$$

where $p_{\text {pos }}^{(k)}$ is the $(x, y)$ position of the drone at time instant $k, w_{\text {pos }}^{(k)}$ is the $(x, y)$ position of the waypoint to reach at time instant $k$, and $N$ is the time instant at which the last waypoint is reached. In addition, the tests consider two levels of accuracy for the positioning error of parked cars (see Section 6.1): full accuracy (i.e. the ideal case) with $\sigma_{P}=0 \mathrm{~m}$, and fair accuracy with $\sigma_{P}=2 \mathrm{~m}$.

The setup used for the waypoint tracking is: 54 waypoints at around every $8 \mathrm{~m}$ along the chosen path, and an acceptable radius of proximity $r_{p}$ of $2 \mathrm{~m}$ to to validate that a given waypoint has been reached. Simulation results for the experiments are presented in Table 3.

Table 3. Simulation results: MAE, traking error $I_{T}$ (Eq. (2)), and travelling time in the drone application.

\begin{tabular}{|c|c|c|c|}
\hline \multirow{2}{*}{} & MAE $[\mathrm{m}]$ & $\begin{array}{c}\text { Tracking } \\
\text { error }\left(I_{T}[\mathrm{~km}]\right)\end{array}$ & $\begin{array}{c}\text { Travelling } \\
\text { time }[\mathrm{min}]\end{array}$ \\
\cline { 2 - 4 } & \multicolumn{3}{|c|}{ GPS positioning } \\
\cline { 2 - 4 } & 7.5636 & 3.2919 & 4.4590 \\
\hline Full accuracy, $\sigma_{P}=0 \mathrm{~m}$ & 0.6206 & 2.2404 & 3.2186 \\
\hline Fair accuracy, $\sigma_{P}=2 \mathrm{~m}$ & 1.8629 & 2.3774 & 3.3995 \\
\hline
\end{tabular}

From in Table 3 it can be observed that the proposed approach delivers a higher performance regarding the MAE, the waypoint tracking error, and the travelling time with respect to GPS positioning. These improvements are of special value in drone navigation, due to some important issues:

- the waypoint tracking is performed by the drone in an autonomous mode (i.e. with no direct human intervention), and thus a smaller positioning error reduces the probability that the drone may hit existing obstacles along the travelled path;

- a more accurate location estimation process allows the drone to reduce the drifting around the recommended path, which in turn reduces the travelling time, which translates into a better exploitation of the drone's flight time autonomy.

\section{Concluding remarks and future work}

Despite the recent advances in the area of localisation techniques for VANETs, there is a shared consensus that the accuracy of conventional (GNSS-based) CP methods is still not good enough for many critical ITS applications, and that there is a general need for V2X-based CP approaches (5). In general, improvements in the performance of CP systems can be achieved not only by designing new localisation algorithms, but also by improving the accuracy of existing methods. In this paper, we followed the second strategy, and showed that by using stationary vehicles to increase the density of highly reliable reference nodes, and by a selection strategy of neighbouring nodes to perform CP estimation, it is possible to greatly enhance the amount and quality of the sources of contextual information, which in turn improves the performance of existing CP algorithms. In particular:

(i) Parked cars augment the density of the fixed roadside-like infrastructure, and so more accurate and reliable contextual (ranging) information is available to moving vehicles. Thus, parked vehicles have the potential to greatly augment any kind of range-based CP algorithms.

(ii) Incorporating parked vehicles as reference nodes into $\mathrm{CP}$ algorithms implies that $\mathrm{CP}$ may 
even be possible when other moving vehicles or dedicated infrastructure are not available to harvest contextual data. This greatly helps not only range-based CP algorithms, but also even some range-free (connectivity-based) CP algorithms such as hop-counting protocols (4).

(iii) As road-side parked vehicles and vehicle stopped in traffic are usually in close proximity to moving cars, communications are expected to be less noisy as the corresponding line-of-sight is less prone to be blocked, thus mitigating the measurement noise produced by shadowing and multi-path effects, this in turn facilitating LOS localisation protocols and existing estimation algorithms for CP (4).

(iv) Finally, localised stationary vehicles can help other stationary vehicles with no position information to localise themselves. Thus, they also improve CP algorithms for static networks.

One specific contribution of this paper, among others, is the evaluation of the use of on-street parked cars as an alternative to RSUs for CP in VANETs. RSUs are, in general, fixed roadside stations that act like beacons or anchors depending on the accuracy with which their position is known (obtained via a dedicated calibration process). Despite the benefits of having a large number of RSUs in the VANETs, their deployment usually requires a complex and expensive process. In this context, our alternative solution shows to be generally cheaper, easier (no complex calibration process is required), and more flexible than fixed infrastructure. Further, as the vehicle fleet refreshes itself after a number of years, technology updates are easier to realise using vehicles as a platform. Also, the proximity of on-street parked cars reduces the requirements on communication ranges, which in turn shortens the time required for accurate localisation of unlocalised cars. Finally, parked cars are dense, thus offering a potential platform for autonomous vehicles in urban situations.

Our simulations demonstrate the potential of this technique in several settings. On-going work involves testing these and related ideas (e.g., a more elaborated selection strategy that takes into account the geographical distribution of neighbouring nodes), in cooperation with a vehicle manufacturer, and using real V2X systems and a real drone.

\section{Acknowledgment}

This work was supported in part by both the Science Foundation Ireland grant $11 / \mathrm{PI} / 1177$, and the European Commission under TEAM, a large scale integrated project part of the FP7-ICT for Cooperative Systems for energy-efficient and sustainable mobility. In addition, the authors would like to thank Wynita M. Griggs for her support and assistance with this research work.

\section{References}

[1] A. Boukerche, H. A. Oliveira, E. F. Nakamura, and A. A. Loureiro, "Vehicular ad hoc networks: A new challenge for localization-based systems," Computer communications, vol. 31, no. 12, pp. 2838-2849, 2008.

[2] A. Eskandarian, Handbook of intelligent vehicles. Springer London, 2012.

[3] L. Hobert, A. Festag, I. Llatser, L. Altomare, F. Visintainer, and A. Kovacs, "Enhancements of v2x communication in support of cooperative autonomous driving," IEEE Communications Magazine, vol. 53, pp. 64-70, Dec 2015.

[4] J. Figueiras and S. Frattasi, Mobile positioning and tracking: from conventional to cooperative techniques. John Wiley \& Sons, 2011.

[5] N. Alam and A. Dempster, "Cooperative Positioning for Vehicular Networks: Facts and Future," IEEE Transactions on Intelligent Transportation Systems, vol. 14, pp. 1708-1717, Dec 2013.

[6] E. C. L. Chan and G. Baciu, Introduction to Wireless Localization: With iPhone SDK Examples, ch. Chapter 9: Differential GPS and Assisted GPS, pp. 157-184. John Wiley \& Sons, Ltd, 2012.

[7] T. Navigation, "Why postprocess GPS data?." On-line white paper, 2004. 
[8] R. Cogill, O. Gallay, W. Griggs, C. Lee, Z. Nabi, R. Ordonez, M. Rufli, R. Shorten, T. Tchrakian, R. Verago, F. Wirth, and S. Zhuk, "Parked cars as a service delivery platform," in 3rd ICCVE'14, (Wien, Austria), 2014.

[9] R. H. Ordóñez-Hurtado and R. N. Shorten, "Using stationary vehicles to enhance cooperative positioning in vehicular ad-hoc networks," in 3rd ICCVE'14, (Wien, Austria), 2014.

[10] M. R. Gholami, M. F. Keskin, S. Gezici, and M. Jansson, "Cooperative positioning in wireless networks," Wiley Encyclopedia of Electrical and Electronics Engineering, 2015.

[11] J. Yao, A. Balaei, M. Hassan, N. Alam, and A. Dempster, "Improving cooperative positioning for vehicular networks," IEEE-TVT, vol. 60, pp. 2810-2823, July 2011.

[12] N. Alam, A. Tabatabaei Balaei, and A. Dempster, "A DSRC Doppler-based cooperative positioning enhancement for vehicular networks with GPS availability," Vehicular Technology, IEEE Transactions on, vol. 60, pp. 4462-4470, Nov 2011.

[13] A. Kealy, N. Alam, M. Efatmaneshnik, C. Toth, A. Dempster, and D. Brzezinska, "Collaborative positioning in GPS-challenged environments," in Earth on the Edge: Science for a Sustainable Planet, pp. 493-500, Springer, 2014.

[14] K. Liu, H. B. Lim, E. Frazzoli, H. Ji, and V. C. Lee, "Improving positioning accuracy using gps pseudorange measurements for cooperative vehicular localization," IEEE Transactions on Vehicular Technology, vol. 63, no. 6, pp. 2544-2556, 2014.

[15] S. Tang, N. Kubo, N. Kawanishi, R. Furukawa, A. Hasegawa, and Y. Takeuchi, "Cooperative relative positioning for intelligent transportation system," International Journal of Intelligent Transportation Systems Research, vol. 13, no. 3, pp. 131-142, 2015.

[16] F. Malandrino, C. Casetti, C. F. Chiasserini, C. Sommer, and F. Dressler, "Content downloading in vehicular networks: Bringing parked cars into the picture," in 23rd IEEE PIMRC'12, pp. 1534-1539, 2012.

[17] N. Liu, M. Liu, G. Chen, and J. Cao, "The sharing at roadside: Vehicular content distribution using parked vehicles," in INFOCOM, 2012 Proceedings IEEE, pp. 2641-2645, 2012.

[18] C. Campolo and A. Molinaro, "DREAM: IEEE 802.11p/WAVE extended access mode in drive-thru vehicular scenarios," in IEEE ICC'12, pp. 5301-5305, June 2012.

[19] C. Campolo and A. Molinaro, "Improving multi-channel operations in VANETs by leveraging stopped vehicles," in 24th IEEE PIMRC'13, pp. 2229-2233, Sept 2013.

[20] C. Sommer, D. Eckhoff, and F. Dressler, "IVC in cities: Signal attenuation by buildings and how parked cars can improve the situation," IEEE Transactions on Mobile Computing, vol. 13, no. 8, pp. 1733-1745, 2014.

[21] O. Tonguz and W. Viriyasitavat, "Cars as roadside units: a self-organizing network solution," Communications Magazine, IEEE, vol. 51, pp. 112-120, December 2013.

[22] A. Reis and S. Sargento, "Leveraging parked cars as urban self-organizing road-side units," in IEEE 82nd Vehicular Technology Conference (VTC2015-Fall), 2015.

[23] J. Kenney, "Dedicated Short-Range Communications (DSRC) Standards in the United States," Proceedings of the IEEE, vol. 99, no. 7, pp. 1162-1182, 2011.

[24] R. Parker and S. Valaee, "Cooperative vehicle position estimation," in IEEE ICC '07, pp. 5837-5842, June 2007.

[25] G. Bellusci, G. J. M. Janssen, J. Yan, and C. C. J. M. Tiberius, "Modeling distance and bandwidth dependency of TOA-based UWB ranging error for positioning," Research Letters in Communications, vol. 2009, p. 4 p., 2009. Article ID 468597.

[26] W. J. Hughes, "Global Positioning System (GPS) Standard Positioning Service (SPS) Performance Analysis Report," tech. rep., Federal Aviation Administration, Washington, DC, 2014.

[27] J. Schipperijn, J. Kerr, S. Duncan, T. Madsen, C. D. Klinker, and J. Troelsen, "Dynamic accuracy of GPS receivers for use in health research: a novel method to assess GPS accuracy in real-world settings," Frontiers in Public Health, pp. 2-21, 2014.

[28] J. Gozalvez, M. Sepulcre, and R. Bauza, "Impact of the radio channel modelling on the performance of VANET communication protocols," Telecommunication Systems, vol. 50, no. 3, pp. 149-167, 2012.

[29] R. Crepaldi, R. Welsh, and R. Kravets, "Governing energy for parked cars," in 10th WONS '13, pp. 8794, 2013.

[30] W. Lin, Y. ru Su, F. Chang, K.-Y. Chen, and S.-H. Huang, "Evaluation approaches of fuel saving and carbon reduction benefits for arterial signal retiming," in 12th ITST '12, pp. 370-374, Nov 2012. 
[31] T. Sando and R. Moses, "Influence of intersection geometrics on the operation of triple left-turn lanes," Journal of transportation engineering, vol. 135, no. 5, pp. 253-259, 2009.

[32] G. Pasaoglu, D. Fiorello, A. Martino, G. Scarcella, A. Alemanno, A. Zubaryeva, and C. Thiel, "Driving and parking patterns of European car drivers -a mobility survey," Research Report JRC77079, Joint Research Centre, European Commission, 2012.

[33] T. Litman, Parking management: strategies, evaluation and planning. Victoria Transport Policy Institute, 2013.

[34] C. Morency and M. Trépanier, "Characterizing parking spaces using travel survey data," tech. rep., CIRRELT, Canada, May 2008.

[35] EPA and I.-U. team, "Scope of Parking in Europe," tech. rep., European Paking Association, http://www.europeanparking.eu/, 2013.

[36] F. Van den Bergh and A. P. Engelbrecht, "A convergence proof for the particle swarm optimiser," Fundamenta Informaticae, vol. 105, no. 4, pp. 341-374, 2010.

[37] S. Zhang, J. Wang, X. Liu, and J. Cao, "Range-free selective multilateration for anisotropic wireless sensor networks," in 9th IEEE SECON'12, pp. 299-307, 2012.

[38] B. W. Tolman, "GPS precise absolute positioning via Kalman filtering," ION GNSS'08, pp. 1864-1874, 2008.

[39] P. Mosterman, D. Sanabria, E. Bilgin, K. Zhang, and J. Zander, "A heterogeneous fleet of vehicles for automated humanitarian missions," Computing in Science Engineering, vol. 16, pp. 90-95, May 2014. 